\title{
¿Vamos hacia una Colombia bilingüe? Análisis de la brecha académica entre el sector público y privado en la educación del inglés
}

Susana Mejía-Mejía

Universidad Eafit, Colombia

smejiame@eafit.edu.co

\section{Resumen}

El trabajo busca explicar los factores que determinan la brecha existente en el nivel de inglés entre los colegios del sector público y los del sector privado, y determinar si esta diferencia se ha reducido en el tiempo con la implementación de políticas públicas orientadas a la generalización del bilingüismo en el país. Para esto, se analizaron los resultados en idiomas de las pruebas Saber 11 en los años 2008 y 2013. Se establece una función de producción educativa que involucra factores familiares, personales $y$ escolares, y una aproximación al componente no observable de la habilidad. La diferencia de rendimiento se ha mantenido constante, aunque con una ligera disminución; sin embargo, se encuentra que la parte no explicada, que puede atribuirse al componente motivacional de los estudiantes, se ha reducido considerablemente, lo que indica que las politicas públicas están sirviendo para que los estudiantes se interesen en el aprendizaje de una lengua extranjera.

\section{Palabras clave}

Evaluación de politicas educativas; Colombia bilingüe; enseñanza del inglés; rendimiento escolar; evaluación educación media (Fuente: Tesauro de la Unesco).

Recepción: 20/12/2014 | Envío a pares: 5/02/2015 | Aceptación por pares: 17/03/2016 | Aprobación: 17/04/2016 DOI: 10.5294/edu.2016.19.2.3

Para citar este artículo / To reference this article / Para citar este artigo

Mejía Mejía, S. (2016). ¿Vamos hacia una Colombia bilingüe? Análisis de la brecha académica entre el sector público y privado en la educación del inglés. Educ. Educ., 19(2), 223-237. DOI: 10.5294/edu.2016.19.2.3 


\title{
Is Colombia Becoming Bilingual?
} Analysis of the Academic Gap in English Language Education between the Public and Private Sectors

\begin{abstract}
The aim of this paper is to explain the factors that determine the gap in the level of English language proficiency between public and private schools, and to determine whether it has narrowed over time as a result of public policies to generalize bilingualism in Colombia. The language scores on the Saber 11 tests for 2008 and 2013 were analyzed for this purpose. An educational production function that involves family, personal and school factors and an approach to the unobservable component of the skill is introduced. The difference in performance has remained more or less constant, but with a slight reduction; however, the unexplained portion, attributable to the students' motivational component, was found to have declined considerably, indicating that public policies are working in terms of helping students to become interested in learning a foreign language.
\end{abstract}

\section{Keywords}

Evaluation of educational policies, bilingual Colombia, teaching English, performance in school, evaluating secondary education (Source: Unesco Thesaurus). 


\section{Rumo a uma Colômbia bilíngue? Análise da brecha acadêmica entre o setor público e o privado na educação de inglês}

\section{Resumo}

Este trabalho pretende explicar os fatores que determinam a brecha existente no nivel de inglês entre os colégios do setor público e os do setor privado, e determinar se essa diferença tem se reduzido no tempo com a implantação de politicas públicas orientadas à generalização do bilinguismo no país. Para isso, analisaram-se os resultados em idiomas das provas Saber 11 em 2008 e 2013. Estabelece-se uma função de produção educativa que envolve fatores familiares, pessoais e escolares, e uma aproximação ao componente não observável da habilidade. A diferença de rendimento tem se mantido constante, embora com uma leve diminuição; contudo, constata-se que a parte não explicada, que pode ser atribuída ao componente motivacional dos estudantes, tem se reduzido consideravelmente, o que indica que as politicas públicas estão servindo para os estudantes se interessem na aprendizagem de uma língua estrangeira.

\section{Palavras-chave}

Avaliação de politicas educativas, Colômbia bilíngue, ensino de inglês, rendimento escolar, avaliação do ensino médio (Fonte: Tesauro da Unesco). 


\section{Introducción}

Desde mediados del siglo XX, con la apertura comercial, la introducción del Internet y la concepción del término "globalización", añadido a la hegemonía de Inglaterra y Estados Unidos, debido a la extensa influencia que tenía el país británico alrededor del mundo y la consolidación de Estados Unidos como primera potencia mundial, tras las crisis en Europa a principios de siglo, el inglés dejó de ser un lenguaje más y pasó a ser visto como una herramienta de trabajo (Casuso, 2014). Tras catalogarse como lengua franca' (Sánchez, 2013), ser declarado como lengua oficial de importantes y numerosas organizaciones internacionales, y conocerse alrededor del mundo como el idioma universal, el estudio del inglés ha dejado de ser una opción, para convertirse en una clara necesidad: fortalecer el dominio de un idioma extranjero es esencial para cualquier sociedad que esté interesada en participar en las dinámicas globales de tipo académico, cultural o económico (MEN, 2014).

Algunas investigaciones han probado que las habilidades lingüísticas pueden ser vistas como componentes del capital humano (Sánchez, 2013); además, en un mundo globalizado como el actual, mejorar los niveles de desempeño en idiomas extranjeros permite el surgimiento de oportunidades, reconocimiento internacional, crecimiento individual y colectivo y mejora las posibilidades de movilidad social (MEN, 2014). Claramente, para ser competitivo en el mercado internacional, un país debe poder desenvolverse bien en el lenguaje en el que se hagan las transacciones; por esto, cada vez es más frecuente que los países aumenten la participación del presupuesto asignado a estrategias diseñadas para mejorar el nivel de sus habitantes en otras lenguas, especialmente en materia de inglés, que se ha denominado el idioma de los negocios.

Estudios recientes han reflejado que el nivel de inglés es muy bajo en Colombia, ubicándose en

1 Idioma común entre personas con diferentes lenguajes maternos (Sánchez, 2013). el último lugar en América Latina (El Tiempo, 2013) y mostrando que el problema tiene raíces profundas debido a falencias en el sistema educativo, la escasez de profesores preparados y la calidad de la enseñanza en las escuelas públicas, que no han sabido encontrar metodologías ni estrategias para motivar a los estudiantes (Sánchez, 2012). Hoy en día, la mayoría de los estudiantes colombianos tiene un nivel de inglés de A- en el Marco Común Europeo de Referencias para Lenguas, que se refiere a que el estudiante no alcanza a tener un conocimiento básico del inglés, y casi el $40 \%$ de los docentes no alcanza a tener un nivel B1 que es la categoría que reconoce a las personas como usuarios independientes del idioma (Sánchez, 2013).

Desde la apertura comercial en 1990, el país ha generado una serie de reformas estratégicas y legislativas para mejorar el estudio del inglés en todos los ámbitos educativos. Es por esto que en 1994, en la Ley General de Educación el Gobierno declaró que el estudio de un idioma extranjero era obligatorio en la formación de los estudiantes de educación básica y media (Ley 115 de 1994), en 2004, el Ministerio de Educación lanzó un proyecto estratégico para el mejoramiento de la calidad de la enseñanza del inglés y la promoción de la competitividad denominado Programa Nacional de Bilingüismo 2004-2019, cuyo objetivo principal es "[...] tener ciudadanos y ciudadanas capaces de comunicarse en inglés, con estándares internacionalmente comparables, que inserten al país en los procesos de comunicación universal, en la economía global y en la apertura cultural" (MEN, 2014). En 2007, los exámenes estatales definieron el inglés como área de evaluación obligatoria (ICFES, 2013) e introdujeron el Marco Común Europeo de Referencia para Lenguas (Sánchez, 2013) para evaluar a los estudiantes con la misma medida que se usa en otras pruebas de carácter internacional, impulsando así las estrategias nacionales y regionales que se venían implementando y permitiendo una comparación con otros países de la región para diagnosticar las competencias lin- 
güísticas. En 2013, se introduce la Ley de Bilingüismo (Ley 1651 de 2013) que agrega regulaciones para la enseñanza del inglés a la Ley General de Educación, y a partir de 2015 comenzó a funcionar el programa Colombia Very Well para mejorar la calidad educativa (El Universal, 2014). A partir de este marco estratégico encuadrado dentro del Proyecto de Fortalecimiento al Desarrollo de Competencias de Lenguas Extranjeras (El Universal, 2014), las alcaldías y los gobiernos regionales se han enfocado en diseñar estrategias que apoyen los planes.

La figura 1 presenta la evolución del puntaje medio en idiomas ${ }^{2}$ en la primera década del 2000, donde se hace evidente que hay una brecha entre las instituciones oficiales y las que no son oficiales. La pregunta que se busca resolver en esta investigación es, dadas las estrategias que se han implementado y los estudios que demuestran que el foco de atención debe ser en los establecimientos educativos oficiales, ¿se ha reducido la brecha en la enseñanza del inglés entre las escuelas públicas y privadas? ¿Están funcionando estas políticas que se han planteado o deben redefinirse otras nuevas? Para responderla, se usa la metodología de descomposición de Oaxaca-Blinder planteando una función de producción educativa y tomando datos de las pruebas estatales Saber 11 para dos años —2008 y 2013-, de modo que se pueda determinar si esta brecha se ha reducido.

Desde la aparición de la teoría del capital humano, que postulaba la educación como factor determinante en el crecimiento económico, los determinantes del rendimiento académico se han convertido en uno de los principales temas de estudio por parte de los investigadores.

Los primeros teóricos en estudiar el impacto del capital humano en el crecimiento económico

2 Antes del 2007, el idioma era flexible y se podía elegir si presentar la prueba de inglés, alemán o francés. Solo a partir del 2007, la prueba del inglés entra a hacer parte de las disciplinas obligatorias (ICFES, 2013).
Figura 1. Puntaje medio en idiomas según tipo de colegio

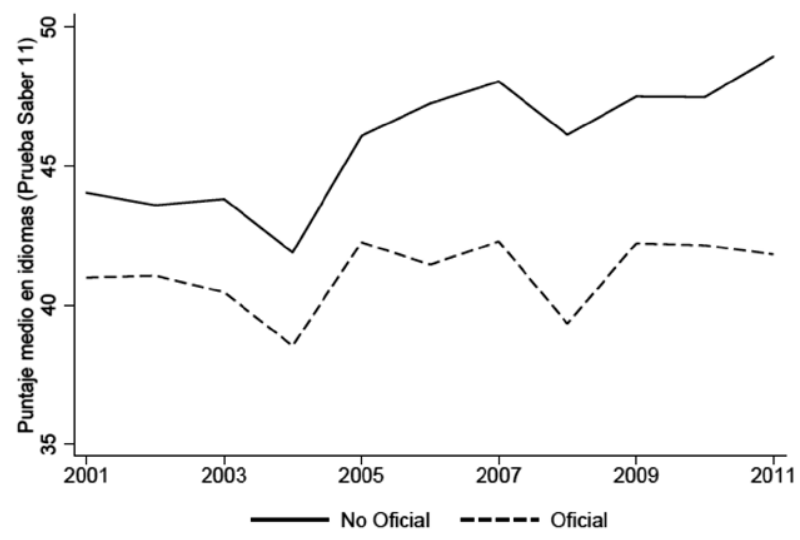

Fuente: Sánchez (2013).

fueron Schultz (1960), Becker (1964) y Mincer (1974), quienes comprobaron la importancia que tenían la salud, la educación y la experiencia en el progreso económico y desarrollaron el primer modelo que relaciona los años de educación con los ingresos, este modelo fue conocido como Ecuación de Mincer (Burgos, 2011). A partir de allí, numerosos estudios han demostrado que la relación entre educación e ingresos es muy estrecha probando que, si existe brecha educativa, por consiguiente existirá brecha salarial (Burgos, 2011); además, a pesar de las críticas que se hacen a las pruebas estatales estandarizadas acerca de su capacidad para medir a los estudiantes, se ha encontrado una relación a largo plazo entre el rendimiento en estas pruebas de diagnóstico y las tasas de crecimiento económico de las regiones (García y Robles, 2012).

Las investigaciones en temas de educación pueden dividirse en tres grandes ramas: en primer lugar están los estudios que se han enfocado en definir los factores determinantes de la educación; luego, aparecen los que buscan definir, a partir de los factores encontrados, la forma funcional de la función de producción educativa y, finalmente, aquellos que se han empeñado en describir las diferencias entre grupos socioeconómicos para encontrar las brechas 
y analizar la forma en que se transmiten las desigualdades (Burgos, 2011).

A partir de las investigaciones de Carroll (1963), quien postuló que el rendimiento académico es un bien producido en el sistema educativo y puede ser medido con una función de producción educativa (Burgos, 2011), los investigadores han clasificado los factores encontrados en tres categorías claves que determinan el rendimiento académico de las personas: las condiciones personales (edad, género, religión), las condiciones familiares (educación de los padres, nivel socioeconómico, número de personas en la casa) y el entorno escolar en el que se desenvuelve el individuo (tipo de colegio, jornada, población académica, calendario). Adicionalmente, varios estudios han definido un cuarto factor no observable, donde se listaría la habilidad innata o la motivación del estudiante, ambas variables difíciles de medir (Hanushek, 1986). Las líneas de investigación se han concentrado en estimar diferentes formas funcionales de la función de producción educativa para determinar cuál es el factor con mayor influencia para explicar el rendimiento escolar. Muchos estudios han probado la importancia que tienen las condiciones familiares sobre los demás factores, lo que demuestra que son las que más afectan el rendimiento del estudiante (Ayala, Marrugo y Saray 2011; Gaviria y Barrientos, 2001; Sánchez, 2011).

Por otro lado, encontrar los factores que determinan las diferencias entre grupos sociales es un tema que se ha tratado ampliamente en la literatura nacional e internacional debido a la importancia que tiene estudiar las brechas para analizar la transmisión de las desigualdades socioeconómicas y frenar la inercia (Burgos, 2011). En Colombia, país de contrastes, se han encontrado brechas académicas a nivel departamental y regional (Baron, 2010), de género (Cárcamo y Mola, 2012), según el tipo de escuela: pública y privada (Castro, Jiménez y Pérez, 2014), por zona rural y urbana (Burgos, 2011), por raza (Sánchez, 2011), por jornada escolar (Bonilla, 2011), entre otras, encontrando resultados interesantes que deberían ser tenidos en cuenta por los hacedores de política para mejorar la calidad educativa en el país y generar movilidad social intergeneracional, rompiendo las trampas de pobreza en las que caen los hogares por la incapacidad de acumular capital humano (Cárdenas, 2007).

Sin embargo, en temas de brecha en la calidad de la educación de segunda lengua no hay literatura existente, las investigaciones que se encuentran de la materia son por lo general cualitativas (Sánchez, 2013) y han buscado revisar los puntos a favor que han tenido las estrategias implementadas y los retos a los que se enfrenta el modelo estratégico para alcanzar la meta de ser un país con alto nivel de inglés en el 2019 (Fandiño, Bérmudez y Lugo, 2012). Por ello, tener un modelo que permita determinar qué factores explican las diferencias en el nivel de inglés entre diferentes grupos sociales puede aportar una idea de qué estrategias adicionales por sector se deben definir para alcanzar la meta y permitir que la población adquiera un mayor nivel de competitividad.

El presente trabajo consta de cinco secciones, además de ésta introducción. En la sección dos se hace una breve exploración metodológica sobre el método de descomposición de Oaxaca-Blinder y se describe la forma como se conformó la base de datos con la que se trabajó. La sección tres plantea la ecuación de producción educativa para explicar el rendimiento de los estudiantes en inglés y los resultados del modelo de descomposición. En la sección cuatro se presenta una discusión de los resultados, y en la sección cinco se presentan las conclusiones y el trabajo futuro.

\section{Metodología}

\section{Descomposición de Oaxaca-Blinder}

El método de descomposición de Oaxaca-Blinder se basa en los trabajos sobre brecha salarial propuestos por Oaxaca (1973) y Blinder (1973). Esta herramienta sirve para cuantificar qué porcentaje de la variable 
es explicada por los factores conocidos y qué se debe a características desconocidas (Burgos, 2011).

Aunque esta metodología ha sido utilizada principalmente para explicar la discriminación en contextos laborales, puede ser usada para describir otro tipo de brechas en variables de resultado (Sánchez, 2011).

El método se presentó inicialmente para modelos lineales y se han hecho desarrollos posteriores para extenderlo a otro tipo de modelos tales como modelos de variable dependiente binaria (Logit, Tobit, Probit); modelos de variable dependiente categórica, donde la variable que se quiere explicar se mide como una variable de satisfacción o felicidad; modelos de regresión no lineales como la regresión cuantílica o la regresión truncada, entre otros.

La ecuación que representa la descomposición para los dos grupos $i=\{O, N\}$ que se están evaluando en este trabajo, donde $O$ se entiende como institución educativa oficial y $N$ como institución educativa no oficial, es:

$\bar{Y}_{O}-\bar{Y}_{N}=\left[\left(\hat{\alpha}_{O}-\hat{\alpha}_{N}\right)+\bar{X}_{O}\left(\hat{\beta}_{O}-\hat{\beta}_{N}\right)\right]+\left[\left(\bar{X}_{O}-\bar{X}_{N}\right) \hat{\beta}_{N}\right]$

donde:

$\bar{Y}_{i}$ es la media del puntaje de inglés de los estudiantes que pertenecen al segmento $i$.

$\bar{X}_{i}$ es la media de las variables que afectan el rendimiento en la educación del inglés de los estudiantes que pertenecen al segmento $i$ y fueron presentados en la sección uno.

$\hat{\alpha}_{i}$ y $\hat{\beta}_{i}$ son los coeficientes estimados del modelo lineal para el segmento $i$.

El lado izquierdo de la ecuación (1) se define como "el gap" o brecha entre los dos grupos que se están evaluando, la primera parte de la ecuación (1) corresponde al componente desconocido, que no puede ser explicado con los factores $\bar{X}_{i}$ y que en la literatura tradicional de mercado laboral se conoce como factor discriminante. La segunda parte muestra el componente que sí es explicado por las variables exógenas. A grandes rasgos, el método realiza una regresión para cada uno de los grupos que se quieren evaluar y calcula la diferencia entre ambos descomponiéndola como se presenta en (1).

\section{Los datos}

La prueba Saber 11 es un examen de Estado que se aplica en Colombia desde la década de los ochenta, evalúa a los estudiantes cuando terminan la educación media y su presentación es obligatoria para continuar con estudios de educación superior. Desde sus comienzos, la prueba ha sufrido dos grandes reformas: en el 2000, dejó de estar centrada en la memorización de contenidos y pasó a ser una prueba que evaluaba por competencias, buscando medir en realidad las capacidades de los estudiantes; a partir del 2007, al núcleo de materias obligatorio se agrega la presentación de la prueba de inglés que, hasta esa fecha, era electiva entre tres opciones de idiomas: inglés, alemán y francés (ICFES, 2013).

Para el presente trabajo se tomaron los datos correspondientes a las pruebas Saber 11 del segundo semestre de los años 2008 y 2013, debido al cambio estructural que se hizo en la prueba a partir del año 2007. Al tomar datos de puntajes en inglés de años después de 2007, se asegura que el idioma que se está evaluando es el inglés y no el francés ni el alemán, además, se tomaron los datos del segundo semestre ya que varias investigaciones explican que a este examen se presenta una cantidad mayor de personas y en el examen del primer semestre por lo general se presentan los colegios bilingües, calendario $B$, con grandes influencias de colegios de habla inglesa asociados en otras partes del mundo (Sánchez, 2013).

La base original constaba de 63 variables que explicaban factores socioeconómicos, personales, familiares y escolares, y 984.817 registros (437.532 correspondientes al año 2008 y 547.285 correspondientes 
al 2013). Para hacer que la base de datos fuera trabajable, se redujo el número de variables a 25 y se tomaron solo los datos de los estudiantes cuyo país de residencia fuera Colombia, que no tuvieran discapacidad y que el municipio en el que vivieran se pudiera clasificar como rural o urbano en una escala de 1 a 5, siguiendo la clasificación propuesta en Posada (2010). Finalmente, al eliminar las observaciones con datos atípicos y datos faltantes, se construyó la base de datos con la que se trabajó, que constaba de 965.637 registros (431.170 de los cuales correspondían al año 2008 y 534.467 al año 2013).

\section{Resultados}

Tomando como base los tres factores propuestos por Carroll (1963), y buscando aproximar el componente no observable al que se refiere Hanushek (1986), se propone una función de producción educativa lineal para determinar las características que explican el rendimiento académico en inglés:

$Y_{i}=f\left(P_{i}, F_{i}, E_{i}, M_{i}\right)$

donde:

$Y_{i}$ es el puntaje de inglés obtenido en la prueba.

$P_{i}$ son los factores personales: edad, y género.

$F_{i}$ son los factores familiares: nivel de ingreso de la familia, tecnologías a las que puede acceder (Internet o televisión cerrada) y zona de residencia clasificada entre muy rural y muy urbana.

Es importante anotar que, aunque otros estudios han demostrado la importancia de la educación de la madre y el padre en el rendimiento académico, estos tienen una alta correlación con el ingreso familiar, por lo que se toma el nivel de ingreso para evitar problemas de multicolinealidad en la especificación del modelo (1).

$E_{i}$ son los factores escolares: jornada completa o parcial, si el colegio es bilingüe o no, tipo de colegio: público o privado, calendario académico, población educativa, entre otros.
Mison los factores no observables atribuibles a la habilidad.

Siguiendo a Miguel y Kremer (2004), quienes usaron el semestre de nacimiento del estudiante para instrumentalizar la habilidad en la presencia de endogeneidad por variables omitidas, se agregó esta variable al modelo pero no resultó significativa estadísticamente, por lo cual se aproxima la habilidad tomando el puntaje de matemáticas en la misma prueba debido a la percepción de algunos autores de tomar las matemáticas como una rama científica que depende en gran medida de la capacidad y la habilidad innatas. En la tabla 1 se presenta la media y la varianza de las diferentes variables explicativas en cada año.

En la tabla 2 se muestra la media y la varianza del puntaje en idiomas consolidado y según la naturaleza del establecimiento educativo, en la última columna se presenta el porcentaje de crecimiento que ha tenido el promedio por segmento entre el 2008 y el 2013, viendo que el sector oficial, aunque sigue estando por debajo del sector no oficial, ha presentado un crecimiento porcentual mayor.

Antes de realizar la descomposición, se estimó una regresión en cada año involucrando todas las variables para determinar cuáles de ellas son relevantes para explicar el nivel de inglés de los estudiantes. Se usó una variable dicótoma para identificar los estudiantes que pertenecen a colegios oficiales y aquellos que pertenecen a los no oficiales, y determinar así si las diferencias son significativas y las variables son adecuadas para explicar dichas diferencias. Las variables categóricas se escribieron como variables binarias para definir si tenían impacto en el modelo.

La tabla 3 presenta los resultados de ambas regresiones; se observa que en el 2008 todas las variables son estadísticamente significativas, por lo que los factores descritos anteriormente son buenos para explicar los resultados en la prueba de inglés. 
Tabla 1. Estadisticas descriptivas de las variables explicativas

\begin{tabular}{|c|c|c|c|c|c|c|}
\hline \multirow{2}{*}{ Factores } & \multirow{2}{*}{ Variable } & \multirow{2}{*}{ Categorías } & \multicolumn{2}{|c|}{2008} & \multicolumn{2}{|c|}{2013} \\
\hline & & & Media & D.S & Media & D.S \\
\hline \multirow{2}{*}{ Personales } & Edad & & 17,361 & 2,842 & 17,186 & 2,757 \\
\hline & Hombre & Hombre & 0,457 & 0,498 & 0,452 & 0,498 \\
\hline \multirow{13}{*}{ Escolar } & Naturaleza & Oficial & 0,744 & 0,436 & 0,753 & 0,431 \\
\hline & Bilingüe & & 0,007 & 0,085 & 0,009 & 0,096 \\
\hline & \multirow{5}{*}{ Jornada } & Completa & 0,205 & 0,404 & 0,195 & 0,397 \\
\hline & & Matutina & 0,506 & 0,500 & 0,519 & 0,500 \\
\hline & & Nocturna & 0,064 & 0,245 & 0,068 & 0,252 \\
\hline & & Sabatina & 0,034 & 0,181 & 0,054 & 0,225 \\
\hline & & Vespertina & 0,191 & 0,393 & 0,164 & 0,370 \\
\hline & \multirow{3}{*}{$\begin{array}{l}\text { Calendario } \\
\text { académico }\end{array}$} & A (Ene-Dic) & 0,877 & 0,329 & 0,847 & 0,360 \\
\hline & & B (Ago-Jun) & 0,001 & 0,025 & 0,004 & 0,062 \\
\hline & & F (Flexible) & 0,123 & 0,328 & 0,149 & 0,356 \\
\hline & \multirow{3}{*}{$\begin{array}{c}\text { Población } \\
\text { escolar }\end{array}$} & Femenino & 0,058 & 0,235 & 0,048 & 0,214 \\
\hline & & Masculino & 0,015 & 0,122 & 0,012 & 0,107 \\
\hline & & Mixto & 0,927 & 0,261 & 0,940 & 0,237 \\
\hline \multirow{14}{*}{ Familiar } & \multirow{5}{*}{$\begin{array}{c}\text { Zona } \\
\text { residencial }\end{array}$} & Muy Rural & 0,018 & 0,133 & 0,019 & 0,136 \\
\hline & & Rural & 0,803 & 0,398 & 0,790 & 0,407 \\
\hline & & Urbano-Rural & 0,034 & 0,180 & 0,035 & 0,184 \\
\hline & & Urbano & 0,052 & 0,222 & 0,058 & 0,234 \\
\hline & & Muy Urbano & 0,094 & 0,292 & 0,098 & 0,297 \\
\hline & \multirow{7}{*}{$\begin{array}{c}\text { Ingreso } \\
\text { familiar } \\
\text { (Según Salario } \\
\text { Mínimo) }\end{array}$} & $<1 S M$ & 0,278 & 0,448 & 0,318 & 0,466 \\
\hline & & $1 S M-2 S M$ & 0,437 & 0,496 & 0,434 & 0,496 \\
\hline & & $2 S M-3 S M$ & 0,163 & 0,370 & 0,141 & 0,348 \\
\hline & & $3 S M-5 S M$ & 0,077 & 0,266 & 0,066 & 0,248 \\
\hline & & $5 S M-7 S M$ & 0,023 & 0,151 & 0,021 & 0,145 \\
\hline & & $7 S M-10 S M$ & 0,011 & 0,106 & 0,011 & 0,103 \\
\hline & & $>10 \mathrm{SM}$ & 0,010 & 0,100 & 0,010 & 0,097 \\
\hline & \multirow{2}{*}{$\begin{array}{c}\text { Acceso a } \\
\text { tecnologías }\end{array}$} & Internet & 0,218 & 0,413 & 0,479 & 0,500 \\
\hline & & TV Cerrada & 0,528 & 0,499 & 0,627 & 0,484 \\
\hline Habilidad & Matemáticas & & 44,512 & 8,139 & 44,849 & 10,244 \\
\hline
\end{tabular}


ISSN $0123-1294$ | Educ.Educ. Vol. 19. No. 2 | Mayo-Agosto de 2016 | pp. 223-237.

Universidad de La Sabana | Facultad de Educación

Tabla 2. Media del puntaje de inglés según naturaleza del colegio por año

\begin{tabular}{|c|c|c|c|c|c|c|}
\hline \multicolumn{2}{|c|}{ Variable } & \multicolumn{2}{c|}{2008} & \multicolumn{2}{c|}{2013} & Crecimiento \\
\hline \multirow{3}{*}{$\begin{array}{c}\text { Puntaje del } \\
\text { inglés }\end{array}$} & Conjunto & 40,83 & {$[30,80,50,85]$} & 44,29 & {$[34,13,54,45]$} & $8,48 \%$ \\
\cline { 2 - 7 } & Oficial & 39,08 & {$[31,77,46,39]$} & 42,70 & {$[34,99,50,42]$} & $9,27 \%$ \\
\cline { 2 - 7 } & No oficial & 45,90 & {$[31,66,60,15]$} & 49,12 & {$[34,79,63,46]$} & $7,01 \%$ \\
\hline
\end{tabular}

Tabla 3. Regresión conjunta de la ecuación (2) para cada año de análisis

\begin{tabular}{|c|c|c|c|c|c|}
\hline Variable & $\mathbf{2 0 0 8}$ & $\mathbf{2 0 1 3}$ & Variable & $\mathbf{2 0 0 8}$ & $\mathbf{2 0 1 3}$ \\
\hline Constante & $32,033(0,164)^{*}$ & $35,056(0,141)^{*}$ & Internet & $2,654(0,038)^{*}$ & $1,067(0,027)^{*}$ \\
\hline Edad & $-0,154(0,005)^{*}$ & $-0,171(0,005)^{*}$ & TV Cerrada & $0,337(0,028)^{*}$ & $0,304(0,026)^{*}$ \\
\hline Hombre & $0,538(0,026)^{*}$ & $-0,695(0,023)^{*}$ & Matutina & $-1,429(0,037)^{*}$ & $-1,641(0,033)^{*}$ \\
\hline 1 SM - 2 SM & $0,312(0,032)^{*}$ & $0,296(0,028)^{*}$ & Nocturna & $-1,044(0,087)^{*}$ & $-1,546(0,086)^{*}$ \\
\hline 2 SM - 3 SM & $1,231(0,043)^{*}$ & $1,582(0,04)^{*}$ & Sabatina & $-1,748(0,098)^{*}$ & $-1,85(0,087)^{*}$ \\
\hline 3 SM - 5 SM & $3,852(0,058)^{*}$ & $4,421(0,053)^{*}$ & Vespertina & $-1,743(0,046)^{*}$ & $-1,908(0,042)^{*}$ \\
\hline 5 SM - 7 SM & $8,091(0,093)^{*}$ & $8,417(0,084)^{*}$ & Bilingüe & $2,261(0,147)^{*}$ & $1,595(0,115)^{*}$ \\
\hline 7 SM - 10 SM & $11,78(0,127)^{*}$ & $12,133(0,114)^{*}$ & B (Ago - Jun) & $4,494(0,497)^{*}$ & $-0,71(0,18)^{*}$ \\
\hline$>10$ SM & $16,748(0,134)^{*}$ & $16,845(0,121)^{*}$ & F (Flexible) & $-1,967(0,069)^{*}$ & $-2,08(0,068)^{*}$ \\
\hline Rural & $0,933(0,097)^{*}$ & $0,768(0,083)^{*}$ & Masculino & $1,318(0,117)^{*}$ & $0,191(0,116)^{*}$ \\
\hline Urbano-Rural & $0,404(0,115)^{*}$ & $0,007(0,1)$ & Mixto & $-2,442(0,057)^{*}$ & $-2,630(0,054)^{*}$ \\
\hline Urbano & $0,417(0,108)^{*}$ & $0,048(0,093)$ & $\begin{array}{c}\text { Puntaje } \\
\text { Matemáticas }\end{array}$ & $0,314(0,002)^{*}$ & $0,346(0,001)^{*}$ \\
\hline Muy Urbano & $0,440(0,102)^{*}$ & $0,031(0,088)$ & Naturaleza & $-2,430(0,038)^{*}$ & $-1,741(0,033)^{*}$ \\
\hline
\end{tabular}

*Estadísticamente significativo a un nivel de confianza $\alpha=0,05$.

En el 2013, todas las variables resultan estadísticamente significativas, excepto las categóricas, que recogen información correspondiente a las zonas urbano-rurales, urbanas y eminentemente urbanas, por tanto, se encuentra que el hecho de ser de una zona urbanizada no impacta el rendimiento en el idioma.

Finalmente, las descomposiciones de la brecha en el 2008 y en el 2013 se presentan en la tabla 4. Se puede ver que las diferencias por tipo de colegio son significativas en los dos años; además, la diferencia que se evidenció en el 2013 (6.419) es muy cercana a la evidenciada en el 2008 (6.820), solo se ha reducido en un $6 \%$, por lo que el tamaño de la brecha no ha cambiado en gran medida, sin embargo, sí se observa un cambio grande en el componente no ex- plicado por las variables independientes - el cual se redujo entre el 2008-, que tenía un valor de $21 \%$, y en el 2013 , en donde ya se tenía un valor de $17 \%$.

\section{Discusión de los resultados}

Los resultados encontrados en la sección tres son interesantes desde diferentes perspectivas. En primer lugar, al observar las salidas de los ejercicios econométricos, se puede ver que, en efecto, existe una brecha entre los sectores público y privado en el nivel de inglés, de alrededor de 6,5 puntos y que, a pesar de ver en la tabla 2 que, en general, el nivel de inglés del país ha mejorado y el sector oficial ha presentado un crecimiento más fuerte $(9,27 \%$ contra $7.01 \%$ del sector no oficial) y en la tabla 3, donde 
Tabla 4. Descomposición de la brecha educativa en nivel de inglés entre colegios del sector privado y el sector público para los años 2008 y 2013

\begin{tabular}{|c|c|c|c|c|c|}
\hline $\begin{array}{c}\text { Puntaje en la prueba } \\
\text { de inglés }\end{array}$ & Coeficiente & Error estándar & Estadístico Z & P-Valor & Porcentaje \\
\hline \multicolumn{6}{|c|}{ Descomposición año 2008} \\
\hline \multicolumn{6}{|l|}{ Diferencial } \\
\hline Predicción 1 & 45,902 & 0,043 & 1069,720 & 0,000 & \\
\hline Predicción 2 & 39,082 & 0,013 & 3028,310 & 0,000 & \\
\hline Diferencia & 6,820 & 0,045 & 152,210 & 0,000 & \\
\hline \multicolumn{6}{|l|}{ Descomposición } \\
\hline Componente explicado & 5,416 & 0,035 & 153,990 & 0,000 & 79 \\
\hline Componente no explicado & 1,405 & 0,024 & 57,710 & 0,000 & 21 \\
\hline \multicolumn{6}{|c|}{ Descomposición año 2013} \\
\hline \multicolumn{6}{|l|}{ Diferencial } \\
\hline Predicción 1 & 49,122 & 0,039 & 1244,790 & 0,000 & \\
\hline Predicción 2 & 42,704 & 0,012 & 3510,680 & 0,000 & \\
\hline Diferencia & 6,419 & 0,041 & 155,440 & 0,000 & \\
\hline \multicolumn{6}{|l|}{ Descomposición } \\
\hline Componente explicado & 5,352 & 0,032 & 166,660 & 0,000 & 83 \\
\hline Componente no explicado & 1,067 & 0,022 & 47,440 & 0,000 & 17 \\
\hline
\end{tabular}

se ve que el coeficiente asociado con la naturaleza del colegio fue menor en términos absolutos en 2013 que en 2008, en términos de brecha, esta se ha mantenido relativamente constante con una pequeña disminución de apenas el $6 \%$ en los últimos cinco años. Sin embargo, encontrar que el porcentaje de la diferencia que no está explicado por las variables señaladas se ha reducido de ser el 21\% de la diferencia en 2008 a 17\% en 2013 es un importante resultado para evaluar los programas estratégicos que se están ejecutando. Si se interpretara el componente no explicado como la motivación que tiene el estudiante hacia el aprendizaje del inglés, podemos afirmar que las estrategias que se están desarrollando han logrado captar el interés de los alumnos hacia el aprendizaje de una segunda lengua y, probablemente, con todas las estrategias diseñadas hacia la mejoría de la calidad docente, la motivación de los profesores también esté creciendo, lo que los ha llevado a renovar su gestión.
Al analizar los resultados de la tabla 3, se encuentran algunas relaciones interesantes que podrían servir como punto de partida para nuevas investigaciones; se debe resaltar el papel que tiene el desempeño en matemáticas para explicar el rendimiento en una segunda lengua, lo que demuestra que esta variable puede usarse para aproximar la habilidad innata que tiene el alumno cuando se está evaluando otro tipo de rendimiento académico como variable dependiente; se ve una fuerte relación directa entre el ingreso y el nivel de inglés, demostrando que entre mayor sea el ingreso familiar, más incentivos (como viajes de turismo o posibilidades de estudios en el exterior) tiene el estudiante para estudiar el idioma. También se encuentra una relación positiva entre el puntaje de la prueba y el acceso a Internet en el hogar, lo que podría sugerir, desde la teoría, que contar con esta herramienta en casa motiva al estudiante a ahondar más el inglés dado que la mayor parte de los sitios de Internet están 
escritos en este idioma. Los resultados también muestran que la jornada parcial (matutina, vespertina, nocturna o sabatina) impacta de manera negativa los puntajes de inglés de los estudiantes, esto va en consonancia con los resultados encontrados por Bonilla (2011) que anunciaba que la doble jornada escolar iba en detrimento de la calidad de la educación en Colombia. Adicionalmente, se presentaron dos resultados que convendría revisar más a fondo en otros estudios; en primer lugar, se ve que la variable binaria Hombre resulta significativa en ambos años, pero muestra diferente signo entre el 2008 y el 2013, esto podría significar que se ha evidenciado una mejora significativa de las mujeres en materia de inglés en los últimos años. Lo mismo ocurre con el calendario B, que en el 2008 resultó significativo y positivo mientras en el 2013 aparece con signo negativo y cercano a cero, a pesar de ser significativo; la posible explicación de este fenómeno sería que la mayor parte de los colegios de calendario B, que en el 2008 presentaban la prueba en el segundo semestre y eran bilingües, están pasando a presentarla en el primer semestre, por lo que ya el calendario no presenta suficiente impacto en los resultados del examen. Por último, conviene analizar la legislación de los colegios bilingües ya que, a pesar de ver que el bilingüismo es significativo en los dos años, no genera un impacto tan grande en los resultados.

Los resultados encontrados en la sección tres indican que la función de producción educativa presentada en (2) y basada en los aportes de Carroll (1963) y Hanushek (1986), sirve también para explicar la calidad de la educación en segunda lengua.

Desde la perspectiva política, es recomendable que los gobernantes tengan en cuenta estos resultados y vean la importancia de diseñar nuevas estrategias: a diez años de introducir el Programa Nacional de Bilingüismo, se ve que aún existen diferencias significativas entre el sector público y el privado, que estas diferencias no se han reducido de manera significativa y que el nivel general de inglés en el país no es bueno.Así, en la tabla 2 se observa que un estudiante promedio tiene un nivel de inglés de 44,29 lo cual equivale a un nivel básico o de principiante $\left(A_{1}\right)$ según el Marco Común Europeo de Referencia para Lenguas (Sánchez, 2013); un estudiante promedio de un colegio público tiene un nivel de inglés de 42,70 que corresponde a un nivel A-, lo que significa que no alcanza a catalogarse con un inglés básico, y el 49,12 que obtiene en promedio un estudiante de colegio privado sigue estando en la categoría básica. Está claro que, aunque la brecha se ha reducido, lo ha hecho muy lentamente y la mejora en el nivel de inglés no ha aumentado al ritmo que se requiere para alcanzar la meta impuesta en el 2004 de ser un país con buen nivel de inglés para el 2019, y que los estudiantes próximos a graduarse tengan un nivel B1 según el Marco Común Europeo (Fandiño et al., 2012; Sánchez, 2013).

Fandiño et al. (2012) y Sánchez (2013) explican que uno de los principales aportes del Programa Nacional de Bilingüismo ha sido poder diagnosticar las competencias lingüísticas de estudiantes y profesores en todo el país e introducir un marco de referencia internacional para poder medir la calidad respecto al nivel de otros países similares. Es por esto que, si conocemos la realidad en la que estamos, si sabemos que la calidad docente no es la mejor (Sánchez, 2013) y que falta motivación para la enseñanza y el aprendizaje, tanto por parte de alumnos como de profesores, es posible diseñar nuevas estrategias en conjunto con los diferentes actores para llegar a consensos entre entidades gubernamentales, profesores y estudiantes, de modo que la actitud de los representantes del Gobierno invite a la construcción de proyectos participativos para acelerar el cierre de la brecha, impulsando a los alumnos del sector público a aprender una nueva lengua, demostrando las oportunidades que esto les brinda, explicando términos como movilidad social y ofreciendo incentivos que motiven a estudiantes y profesores a querer aprender y ser mejores cada día. Según Bonilla (2010, citado en Burgos, 2011). 
...Aunque la zona rural está creciendo y desarrollándose más rápido que la zona urbana gracias a la implementación de los programas de acción sobre la oferta educativa en el campo, aún hay mucho por hacer en la consecución de un escenario de convergencia entre ambos sectores a largo plazo y de reducción de la brecha educativa (p.15).

Sin embargo, el panorama está lleno de oportunidades.

\section{Conclusiones}

Se construyó y estimó una función de producción lineal educativa usando variables personales, familiares y escolares para determinar el impacto de estos factores en el desempeño en competencias lingüísticas de los estudiantes; adicionalmente, se utilizó el rendimiento en matemáticas para aproximar las habilidades innatas del alumno, encontrando significancia estadística.
Se realizó una descomposición de Oaxaca-Blinder en dos periodos para determinar la diferencia existente entre los establecimientos públicos y privados; se encontró que, en efecto, existe una brecha académica y esta se ha mantenido relativamente constante en el tiempo, aunque el factor atribuible a la motivación de los estudiantes se ha reducido, por lo que los planes que se han llevado a cabo han elevado el nivel de interés de los alumnos.

Finalmente, se hace un llamado a los entes gubernamentales, nacionales y regionales, para que analicen los resultados que han arrojado las herramientas implementadas hasta ahora, como el Programa Nacional de Bilingüismo o la introducción del Marco Común Europeo de Referencia para Lenguas, que han permitido diagnosticar los puntos débiles del sistema educativo en inglés, tanto en estudiantes como profesores y, con esto, diseñar nuevos programas estratégicos de manera participativa de modo que entre la comunidad académica y el sector público se acelere el cierre de la brecha y se eleve el nivel de inglés, pues es prioritario para elevar la competitividad del país.

\section{Referencias}

Ayala, J., Marrugo, S.y Saray, B. (2011). Antecedentes familiares y rendimiento académico en los colegios oficiales de Cartagena. Economía y Región, 5 (2), 43-85.

Barón, J. (2010). La brecha de rendimiento académico de Barranquilla. Documentos de Trabajo sobre Economía Regional, 137.

Becker, G. S. (1964). Human Capital. New York: Columbia University Press for the National Bureau of Economic Research.

Blinder, A. S. (1973). Wage discrimination: Reduced form and structural estimates. The Journal of Human Resources, 8 (4), 436-455

Bonilla, L. (2010). Movilidad inter-generacional en educación en las ciudades y regiones de Colombia. Documentos de Trabajo sobre Economía Regional, 130.

Bonilla, L. (2011). Doble jornada escolar y la calidad de la educación en Colombia. Documentos de Trabajo sobre Economía Regional, 143 . 
ISSN $0123-1294$ | Educ.Educ. Vol. 19. No. 2 | Mayo-Agosto de 2016 | pp. 223-237.

Universidad de La Sabana | Facultad de Educación

Burgos, J. I. (2011). Brecha educativa entre población ruraly urbana en Colombia, 2007. Tesis de grado sin publicar. Universidad del Valle. Cali: Colombia.

Cárcamo, C. y Mola, J. (2012). Diferencias por sexo en el desempeño académico en Colombia: un análisis regional. Economía y región, 6 (1), 133-169.

Cárdenas, M. (2007). Introducción a la economía colombiana. Bogotá D.C: Fedesarrollo.

Carroll, J. (1963). A model of school learning. Teachers College Record, 64, 723-733.

Castro, G., Giménez, G.y Pérez, D. (2014). El desempeño educativo escolar en Colombia: factores que determinan la diferencia en rendimiento académico entre las escuelas públicas y privadas. XXIII Jornadas de la Asociación de Economía de la Educación, Valencia.

Casuso, G. (2014). ¿Para qué sirve aprender inglés? Recuperado de http://ingles.about.com

El Tiempo (2013). Siete de cada diez profesores de inglés en el país tienen nivel bajo. Recuperado de http://www. eltiempo.com/archivo/documento/CMS-13144584

El Universal (2014). Mineducación anuncia “Colombia Very Well”, estrategia nacional de bilingüismo. Recuperado de http://www.eluniversal.com.co/educacion/mineducacion-anuncia-colombia-very-well-estrategianacional-de-bilinguismo-164634

Fandiño, Y. J., Bérmudez, J. R. y Lugo, V. E. (2012). Retos del Programa Nacional de Bilingüismo. Colombia Bilingüe. Educación y Educadores, 15 (3), 363-381.

García, J. I.y Robles, J. A. (2012). Diferencias regionales en el rendimiento educativo: ¿Qué ha cambiado entre 2009 y 2012? En Pisa: Resolución de problemas de la vida real. España: Instituto Nacional de Evaluación Educativa.

Gaviria, A. y Barrientos, J. H. (2001). Calidad de la educación y rendimiento académico en Bogotá. Medellín: Universidad de Antioquia.

Hanushek, E. A. (1986). The economics of schooling: Production and efficiency in public schools. Journal of Economic Literature, 24 (3), 1141-1177.

ICFES (2013). Guía de acceso a bases de datos ICFES. Recuperado de http://www.icfes.gov.co/investigacion/acceso-a-bases-de-datos

Ley 115 de 1994. Por la cual se expide la ley general de educación. Colombia: Ministerio de Educación.

Ley 1651 de 2013. Por medio de la cual se modifican los artículos 13, 20, 21, 22, 30 y 38 de la Ley 115 de 1994 y se dictan otras disposiciones - Ley de Bilingüismo. Colombia: Ministerio de Educación.

Miguel, E.y Kremer, M. (2004). Worms: Identifying impacts on education and health in the presence of treatment externalities. Econometrica, 72 (1), 159-217. 
Mincer, J. (1974). Schooling, experience and earnings. New York: National.

Ministerio de Educación Nacional (MEN) (2014). Programa Nacional de Bilingüismo. Recuperado de http:// www.colombiaaprende.edu.co/html/productos/1685/article-158720.html

Oaxaca, R. (1973). Male - female wage differentials in urban labor markets. International Economic Review, 14 (3), 693-709.

Posada, G. J. (2010). Agrupación de municipios colombianos según características de ruralidad [Tesis de Maestría sin publicar]. Universidad Nacional de Colombia, Medellín, Colombia.

Sánchez, A. (2011). Etnia y desempeño académico en Colombia. Documentos de Trabajo sobre Economía Regional, 156.

Sánchez, A. (2013). Bilingüismo en Colombia. Documentos de Trabajo sobre Economía Regional, 191.

Sánchez, V. (2012). Colombia, país latinoamericano con nivel de inglés más bajo. Recuperado de http://www. guiaacademica.com/Educacion/personas/cms/colombia/noticias_academicas/2012/ARTICULO-WEBEEE_PAG-12418885.aspx

Schultz, T. W. (1960). Capital formation by education. The Journal of Political Economy, 68 (6), 571-583. 
